PROCEEDINGS OF THE

AMERICAN MATHEMATICAL SOCIETY

Volume 138, Number 10, October 2010, Pages 3393-3403

S 0002-9939(2010)10133-8

Article electronically published on June 3, 2010

\title{
POINCARÉ SERIES AND THE DIVISORS OF MODULAR FORMS
}

\author{
D. CHOI
}

(Communicated by Ken Ono)

\begin{abstract}
Recently, Bruinier, Kohnen and Ono obtained an explicit description of the action of the theta-operator on meromorphic modular forms $f$ on $S L_{2}(\mathbb{Z})$ in terms of the values of modular functions at points in the divisor of $f$. Using this result, they studied the exponents in the infinite product expansion of a modular form and recurrence relations for Fourier coefficients of a modular form. In this paper, we extend these results to meromorphic modular forms on $\Gamma_{0}(N)$ for an arbitrary positive integer $N>1$.
\end{abstract}

\section{INTRODUCTION}

Let $N$ be a positive integer and $q:=e^{2 \pi i z}, z=x+i y$. If $f(z)=\sum_{n=h}^{\infty} a(n) q^{n}$ is a meromorphic modular form on $\Gamma_{0}(N)$, then we define the theta-operator by

$$
\theta f(z):=\frac{1}{2 \pi i} \frac{d}{d z} f(z)=\sum_{n=h}^{\infty} n a(n) q^{n} .
$$

Recently, Bruinier, Kohnen and Ono obtained in [6] an explicit description of the action of the theta-operator on meromorphic modular forms on $S L_{2}(\mathbb{Z})$ in terms of the values of modular functions at points in the divisor of $f$. Using this result, they also studied the exponents in the infinite product expansion of a modular form and recurrence relations for the Fourier coefficients of modular forms (see Theorems 3 and 5 in [6]). Ahlgren gave analogues of these results for meromorphic modular forms on $\Gamma_{0}(p)$ for $p \in\{2,3,5,7,13\}$ (see [2]). For general primes $p$, using eta-quotients, the author studied in 8 , the action of the theta-operator on meromorphic modular forms on $\Gamma_{0}(p)$ and the exponents in their infinite product expansion. But, recurrence relations for the Fourier coefficients of the modular forms in 6 and 2 were not generalized in 8 . In this paper, we extend the results 2 to meromorphic modular forms on $\Gamma_{0}(N)$ for an arbitrary positive integer $N>1$ and obtain recurrence relations for their Fourier coefficients.

To obtain our main theorems, we consider Poincaré series of weight 0 instead of eta-quotients. Since in general these Poincaré series are not meromorphic functions on the complex upper half plane, we cannot use the valence formula or the residue theorem as in [6], [2] and [8]. Thus, following the argument of [5], we use the regularized integral and Stokes' Theorem.

Received by the editors April 2, 2009 and, in revised form, July 27, 2009.

2010 Mathematics Subject Classification. Primary 11F12; Secondary 11F30.

Key words and phrases. Borcherds exponents, Poincaré series, divisors of modular forms.

(C) 2010 American Mathematical Society 
To state our results, we introduce some notation. The group $\Gamma_{0}(N)$ is the congruence subgroup of $S L_{2}(\mathbb{Z})$ defined as

$$
\Gamma_{0}(N)=\left\{\left(\begin{array}{ll}
a & b \\
c & d
\end{array}\right) \in S L_{2}(\mathbb{Z}) \mid c \equiv 0 \quad(\bmod N)\right\} .
$$

Let $\mathcal{C}_{N}$ be the set of inequivalent cusps of $\Gamma_{0}(N)$ and $\mathcal{C}_{N}^{*}=\mathcal{C}_{N} \backslash\{\infty\}$. For a cusp $u$ of $\Gamma_{0}(N)$ let

$$
\Gamma_{0}(N)_{u}:=\left\{\sigma \in \Gamma_{0}(N) \mid \sigma u=u\right\} .
$$

Here, $\sigma u:=\frac{a u+b}{c u+d}$ for $\sigma=\left(\begin{array}{ll}a & b \\ c & d\end{array}\right) \in S L_{2}(\mathbb{R})$. We denote by $\mathcal{F}_{N}$ a fundamental domain for the action of $\Gamma_{0}(N)$ on $\mathbb{H}$. The modular curve $X_{0}(N)$ is defined as the quotient space of orbits under $\Gamma_{0}(N), X_{0}(N)=\Gamma_{0}(N) \backslash \mathbb{H} \cup \mathbb{Q} \cup\{\infty\}$. Suppose that $f(z)$ is a meromorphic modular form of weight $k$ on $\Gamma_{0}(N)$. We consider the following functions: a meromorphic modular form $f_{\theta}(z)$ and a Poincaré series $j_{N, m}(z)$ of weight 0 and index $m$. For $N>1$ let

$$
f_{\theta}(z):=\frac{\theta f(z)}{f(z)}+\frac{k / 12-h}{N-1} \cdot N E_{2}(N z)+\frac{h-N k / 12}{N-1} \cdot E_{2}(z) .
$$

Here, $E_{2}(z)$ is the usual normalized Eisenstein series of weight 2 defined by

$$
E_{2}(z)=1-24 \sum_{n \geq 1} \sigma_{1}(n) q^{n}
$$

where $\sigma_{k}(n):=\sum_{d \mid n} d^{k}$ if $n \in \mathbb{N}$ and $\sigma_{k}(n)=0$ if $n \notin \mathbb{N}$.

Let $I_{v}(z)$ be the usual modified Bessel functions as in [1] and $e(x):=e^{2 \pi i x}$. For a positive integer $m$ we define the Poincaré series of weight 0 and index $m$ by (1.1)

$$
F_{N, m}(z, s):=\sum_{\gamma \in \Gamma_{0}(N)_{\infty} \backslash \Gamma_{0}(N)} \pi|m \operatorname{Im}(\gamma z)|^{1 / 2} I_{s-\frac{1}{2}}(|2 \pi m \operatorname{Im}(\gamma z)|) e(-m \operatorname{Re}(\gamma z))
$$

where $z \in \mathbb{H}$ and $s \in \mathbb{C}$ with $\operatorname{Re}(s)>1$. Let $j_{N, m}(z)$ be the continuation of $F_{N, m}(z, s)$ as $s \rightarrow 1$ from the right. The function $j_{N, m}(z)$ is a weak Maass form on $\Gamma_{0}(N)$ (see Section 2 for details). For convenience, if $t$ is a cusp of $\Gamma_{0}(N)$, then $j_{N, m}(t)$ denotes the constant term of the Fourier expansion of $F_{N, m}(z, 1)$ at $t$. We define a differential operator $\xi_{0}$ by

$$
\xi_{0}\left(j_{N, m}\right)(z):=2 i \overline{\frac{\partial}{\partial \bar{z}} j_{N, m}(z)}
$$

and consider the integral

$$
\int_{\mathcal{F}_{N}} f_{\theta}(z) \cdot \xi_{0}\left(j_{N, m}(z)\right) d x d y
$$

Note that in general $f_{\theta}(z)$ is not holomorphic on $\mathbb{H}$. Thus, we have to regularize the integral (1.3) and denote it by

$$
\int_{\mathcal{F}_{N}}^{r e g} f_{\theta}(z) \cdot \xi_{0}\left(j_{N, m}(z)\right) d x d y
$$

(see (3.1) in Section 3 for the exact definition). With this notation, we state our main theorem. 
Theorem 1.1. Suppose that $f(z):=q^{h}+\sum_{n=h+1}^{\infty} a(n) q^{n}$ is a meromorphic modular form of weight $k$ on $\Gamma_{0}(N)$ with a positive integer $N, N>1$. Let $\{c(n)\}_{n=1}^{\infty}$ be the complex numbers for which

$$
f(z)=q^{h} \prod_{n=1}^{\infty}\left(1-q^{n}\right)^{c(n)} .
$$

Then we have

$$
\begin{aligned}
\sum_{d \mid m} d \cdot c(d) & =\sum_{\tau \in \mathcal{F}_{N} \cup \mathcal{C}_{N}^{*}} \nu_{\tau}^{(N)}(f(z)) j_{N, m}(\tau)-\int_{\mathcal{F}_{N}}^{r e g} f_{\theta}(z) \cdot \xi_{0}\left(j_{N, m}(z)\right) d x d y \\
& +\frac{2 N k-24 h}{N-1} \sigma_{1}(m)+\frac{24 h-2 k}{N-1} N \sigma_{1}(m / N) .
\end{aligned}
$$

Here, $\nu_{\tau}^{(N)}(f(z))$ denotes the order of zero of $f(z)$ at $\tau$ on $X_{0}(N)$.

Remark 1.2. Suppose that $j_{N, m}(z)$ is holomorphic on $\mathbb{H}$. From the definition of the differential operator (1.2) we have

$$
\xi_{0}\left(j_{N, m}(z)\right)=0 .
$$

This implies that in Theorem 1.1 .

$$
\int_{\mathcal{F}_{N}}^{r e g} f_{\theta}(z) \cdot \xi_{0}\left(j_{N, m}(z)\right) d x d y=0 .
$$

Remark 1.3. From Remark 1.2 it may be an interesting question when $j_{N, m}(z)$ is holomorphic on $\mathbb{H}$. Since $\xi_{0}\left(j_{N, m}(z)\right)$ is a cusp form, the results of [5] imply that $j_{N, m}(z)$ is holomorphic on $\mathbb{H}$ if and only if for every cusp form $g(z)=\sum_{n=1}^{\infty} b(n) q^{n}$ of weight 2 on $\Gamma_{0}(N)$ we have

$$
b(m)=0
$$

(1) Suppose that the genus of $\Gamma_{0}(N)$ is zero. Then $j_{N, m}(z)$ is holomorphic on $\mathbb{H}$. Thus, when $N \in\{2,3,5,7,13\}$, Theorem 1.1 recovers the result of Ahlgren [2.

(2) Suppose that the genus of $\Gamma_{0}(N)$ is one and that $g(z)=\sum_{n=1}^{\infty} b(n) q^{n}$ is the unique normalized cusp form of weight 2 on $\Gamma_{0}(N)$. For a prime $p$ let $\mathbf{F}_{p}$ denote a finite field $\mathbb{Z} / p \mathbb{Z}$. There exists an elliptic curve $E_{g}$ of conductor $N^{\prime}, N^{\prime} \mid N$, defined over $\mathbb{Q}$ such that for all $p \nmid N$,

$$
1-a(p)+p=\sharp E_{g}\left(\mathbf{F}_{p}\right) .
$$

Note that $|a(p)| \leq 2 \sqrt{p}$ and $g(z)$ is a Hecke eigenform. Thus, for an odd integer $m, j_{N, m}(z)$ is holomorphic on $\mathbb{H}$ if and only if $E_{g}$ is supersingular at $p$ for some prime $p, p \mid m$. In [10] Elkies proved the existence of infinitely many supersingular primes for every elliptic curve defined over $\mathbb{Q}$. This implies that there are infinitely many primes $p$ such that $j_{N, m}(z)$ is holomorphic on $\mathbb{H}$ for every positive integer $m, p \mid m$.

Using Theorem 1.1, we obtain a description of the action of the theta-operator on meromorphic modular forms on $\Gamma_{0}(N)$. 
Theorem 1.4. Suppose that $f(z):=q^{h}+\sum_{n=h+1}^{\infty} a(n) q^{n}$ is a meromorphic modular form of weight $k$ on $\Gamma_{0}(N)$ with a positive integer $N, N>1$. Then

$$
\begin{aligned}
f_{\theta}(z)+\sum_{m=1}^{\infty}\left(\int_{\mathcal{F}_{N}}^{r e g} f_{\theta}(z) \cdot \xi_{0}\left(j_{N, m}(z)\right) d x d y\right) q^{m} \\
=\sum_{m=1}^{\infty}\left(\sum_{\tau \in \mathcal{F}_{N} \cup \mathcal{C}_{N}^{*}} \nu_{\tau}^{(N)}(f(z)) j_{N, m}(\tau)\right) q^{m} .
\end{aligned}
$$

From Remark 1.3(2) and Theorem 1.4 we have immediately the following corollary.

Corollary 1.5. Suppose that the genus of $\Gamma_{0}(N)$ is one and that $f(z):=q^{h}+$ $\sum_{n=h+1}^{\infty} a(n) q^{n}$ is a meromorphic modular form of weight $k$ on $\Gamma_{0}(N)$ with a positive integer $N, N>1$. Then there are infinitely many primes $p$ such that

$$
f_{\theta}(z) \mid U_{p}=\sum_{m=1}^{\infty}\left(\sum_{\tau \in \mathcal{F}_{N} \cup \mathcal{C}_{N}^{*}} \nu_{\tau}^{(N)}(f(z)) j_{N, p m}(\tau)\right) q^{m} .
$$

Remark 1.6. The formula in Corollary 1.5 does not contain the regularized integral. Thus, if $f_{\theta}(z) \mid U_{p} \cdot E_{\ell-1}$ is a modular form for some prime $\ell$, then we can study congruence for the values of $j_{N, p m}$ by using the argument of [6]. But, it seems difficult to check whether $f_{\theta}(z) \mid U_{p} \cdot E_{\ell-1}$ is a modular form.

As another application of Theorem 1.1 we obtain universal recursion formulas for coefficients of meromorphic modular forms on $\Gamma_{0}(N)$. For each $n \geq 1$, we define the polynomial

$$
\begin{gathered}
F_{n}^{(N)}\left(K, H, x_{1}, \cdots, x_{n}\right):= \\
\sum_{\substack{m_{1}+2 m_{2}+\cdots+(n-1) m_{n-1}=n \\
m_{1}, \cdots, m_{n-1} \geq 0}}(-1)^{m_{1}+\cdots+m_{n-1}} \frac{\left(m_{1}+\cdots+m_{n-1}-1\right) !}{m_{1} ! m_{2} ! \cdots m_{n-1} !} x_{1}^{m_{1}} \cdots x_{n-1}^{m_{n-1}} \\
-\frac{1}{n}\left(\frac{2 N K-24 H}{N-1}\right) \sigma_{1}(m)-\frac{1}{n}\left(\frac{24 H-2 K}{N-1}\right) N \sigma_{1}(m / N) .
\end{gathered}
$$

Theorem 1.7. Suppose that

$$
f(z):=q^{h}+\sum_{n=h+1}^{\infty} a(n) q^{n}
$$

is a meromorphic modular form of weight $k$ on $\Gamma_{0}(N)$ with a positive integer $N$, $N>1$. For each $n \geq 1$, the polynomial $F_{n}^{(N)}\left(K, H, x_{1}, \cdots, x_{n}\right)$ is defined as in (1.5). Then we have

$$
\begin{aligned}
a(h+n) & =F_{n}^{(N)}(k, h, a(h+1), \cdots, a(h+n-1)) \\
& -\frac{1}{n} \sum_{\tau \in \mathcal{F}_{N} \cup \mathcal{C}_{N}^{*}} \nu_{\tau}^{(N)}(f(z)) j_{N, m}(\tau)+\frac{1}{n} \int_{\mathcal{F}_{N}}^{r e g} f_{\theta}(z) \cdot \xi_{0}\left(j_{N, m}(z)\right) d x d y .
\end{aligned}
$$

Example 1.8. Let $E$ be an elliptic curve of conductor $N$ defined over $\mathbb{Q}$ and let $\mathbf{F}_{p}$ denote a finite field $\mathbb{Z} / p \mathbb{Z}$ for a prime $p$. It is known that there is a normalized 
Hecke eigenform $f(z)=\sum_{n=1}^{\infty} a(n) q^{n}$ of weight 2 on $\Gamma_{0}(N)$ such that for all $p \nmid N$,

$$
1-a(p)+p=\sharp E\left(\mathbf{F}_{p}\right) \text {. }
$$

From Theorem 1.7 we have

$$
\begin{aligned}
a(1+n) & =F_{n}^{(N)}(2,1, a(2), \cdots, a(n)) \\
& -\frac{1}{n} \sum_{\tau \in \mathcal{F}_{N} \cup \mathcal{C}_{N}^{*}} \nu_{\tau}^{(N)}(f(z)) j_{N, m}(\tau)+\frac{1}{n} \int_{\mathcal{F}_{N}}^{r e g} f_{\theta}(z) \cdot \xi_{0}\left(j_{N, m}(z)\right) d x d y .
\end{aligned}
$$

By the definition of the Hecke operator, we obtain from (1.6) recursive relations for $\sharp E\left(\mathbf{F}_{p}\right)$. For example, if $N=11$ and $m=1$, then

$$
\int_{\mathcal{F}_{N}}^{r e g} f_{\theta}(z) \cdot \xi_{0}\left(j_{N, m}(z)\right) d x d y=0
$$

Remark 1.9. Our results (Theorems 1.1, 1.4 and 1.7) can be immediately extended to congruence subgroups $\Gamma \subset \Gamma_{0}(N)$ for $N>1$.

This paper is organized as follows. In section 2 we recall the definition of Poincaré series of weight zero on $\Gamma_{0}(N)$. In section 3 we define the regularized integral of a meromorphic modular form of weight 2 . In sections 4 and 5 we give the proofs of the main theorems.

\section{Poincaré series of Weight 0 on $\Gamma_{0}(N)$}

In this section we consider non-holomorphic Poincaré series of weight 0. For details we refer to [11, [13, [4, 7].

Let $I_{v}(z)$ and $K_{v}(z)$ be the usual modified Bessel functions as in [1]. We define for $s \in \mathbb{C}$ and $y \in \mathbb{R} \backslash\{0\}$ :

$$
\begin{aligned}
\mathcal{I}_{s}(y) & :=\sqrt{\frac{\pi|y|}{2}} I_{s-\frac{1}{2}}(|y|), \\
\mathcal{K}_{s}(y) & :=\sqrt{\frac{\pi|y|}{2}} K_{s-\frac{1}{2}}(|y|) .
\end{aligned}
$$

Note that $\mathcal{I}_{s}(y)$ and $\mathcal{K}_{s}(y)$ are holomorphic in $s$. If $s=1$, then we have

$$
\begin{aligned}
\mathcal{I}_{1}(y) & =\sinh (|y|), \\
\mathcal{K}_{1}(y) & =e^{-|y|}, \\
2 \mathcal{I}_{1}(y)+\mathcal{K}_{1}(y) & =e^{|y|} .
\end{aligned}
$$

For a cusp $u$ let $\sigma_{u}$ denote a matrix in $S L_{2}(\mathbb{R})$ such that

$$
\sigma_{u}^{-1} \infty=u \text { and } \sigma_{u} \Gamma_{0}(N)_{u} \sigma_{u}^{-1}=\left\{ \pm\left(\begin{array}{cc}
1 & n \\
0 & 1
\end{array}\right) \mid n \in \mathbb{Z}\right\} .
$$

If $t_{1}$ and $t_{2}$ are equivalent cusps of $\Gamma_{0}(N)$, i.e., $\gamma t_{1}=t_{2}$ for some $\gamma \in \Gamma_{0}(N)$, then we write $t_{1} \sim t_{2}$. For a positive integer $m$ we define the Poincaré series of weight 0 and index $m$ by

$$
F_{N, m}(z, s):=\sum_{\left.\gamma \in \Gamma_{0}(N)\right)_{\infty} \backslash \Gamma_{0}(N)} \mathcal{I}_{s}(2 \pi m \operatorname{Im}(\gamma z)) e(-m \operatorname{Re}(\gamma z)),
$$

where $z \in \mathbb{H}$ and $s \in \mathbb{C}$ with $\operatorname{Re}(s)>1$. The special value $F_{N, m}(z, 1)$ satisfies the following properties. 
Theorem 2.1. The function $F_{N, m}(z, 1)$ is a harmonic weak Maass form of weight 0 on $\Gamma_{0}(N)$. Moreover, $F_{N, m}(z, 1)$ has the following properties at each cusp $t$ :

$$
\begin{aligned}
& F_{N, m}(z, 1)=q^{-m}+\sum_{n \geq 0} b_{m}(n, 1) q^{n}+\sum_{n>0} b_{m}(-n, 1) e(n \bar{z}) \text { if } t \sim \infty, \\
& \lim _{\operatorname{Im} z \infty} F_{N, m}\left(\sigma_{t} z, 1\right)=j_{N, m}(t) \quad \text { if } t \nsim \infty .
\end{aligned}
$$

Proof. Let

$$
I_{\gamma}(z, s):=\sum_{\beta \in \Gamma_{0}(N)_{\infty}} \mathcal{I}_{s}(2 \pi m \operatorname{Im}(\gamma \beta z)) e(-m \operatorname{Re}(\gamma \beta z)) .
$$

If we define

$$
\begin{aligned}
& \delta_{t \infty}:=1 \text { if } t \sim \infty \\
& \delta_{t \infty}:=0 \text { if } t \nsim \infty,
\end{aligned}
$$

then

$$
\begin{aligned}
F_{N, m}\left(\sigma_{t} z, s\right)=\delta_{t \infty} \cdot \mathcal{I}_{s}(2 \pi m & \operatorname{Im}(z)) e(-m \operatorname{Re}(z)) \\
& +\sum_{\gamma \in \Gamma_{0}(N)_{\infty} \backslash \Gamma_{0}(N) \sigma_{t} / \Gamma_{0}(N)_{\infty}} \mathcal{I}_{\gamma}(z, s) .
\end{aligned}
$$

Considering the Fourier expansion of

$$
\sum_{\gamma \in \Gamma_{0}(N)_{\infty} \backslash \Gamma_{0}(N) \sigma_{t} / \Gamma_{0}(N)_{\infty}} \mathcal{I}_{\gamma}(z, s)
$$

we can obtain (2.2) and (2.3). For the details of the proof, see $\S 1.9$ in [4] or [1], 13.

\section{Regularized integration}

Suppose that $g(z)$ is a meromorphic modular form of weight 2 on $\Gamma_{0}(N)$. Let $S(g)$ be the set of the singular points of $g(z)$ on $\mathcal{F}_{N}$. We define an $\varepsilon$-disk $B(t, \varepsilon)$ at $t$ by

$$
B(t, \varepsilon):= \begin{cases}\{z \in \mathbb{H}|| z-t \mid<\varepsilon\} & \text { if } t \in \mathcal{F}_{N}, \\ \left\{z \in \mathcal{F}_{N} \mid \operatorname{Im}\left(\sigma_{t} z\right)>\frac{1}{\varepsilon}\right\} & \text { if } t \in \mathcal{C}_{N} .\end{cases}
$$

For sufficiently small positive $\varepsilon$, let $\mathcal{F}_{N}(g, \varepsilon)$ denote a punctured fundamental domain for $\Gamma_{0}(N)$ defined as

$$
\mathcal{F}_{N}(g, \varepsilon)=\mathcal{F}_{N}-\bigcup_{t \in S(g) \cup \mathcal{C}_{N}} B(t, \varepsilon) .
$$

We define the regularized integral of $g(z) \cdot \xi_{0}\left(j_{N, m}(z)\right) d x d y$ on $\mathcal{F}_{N}$ by

$$
\int_{\mathcal{F}_{N}}^{r e g} g(z) \cdot \xi_{0}\left(j_{N, m}(z)\right) d x d y:=\lim _{\varepsilon \rightarrow 0} \int_{\mathcal{F}_{N}(g, \varepsilon)} g(z) \cdot \xi_{0}\left(j_{N, m}(z)\right) d x d y .
$$

Note that $g(z) \cdot \overline{j_{N, m}(z)} d z$ is a $\Gamma_{0}(N)$-invariant 1-form on $\mathbb{H}$. We define

$$
\gamma(t, \varepsilon):= \begin{cases}\{z \in \mathbb{H}|| z-t \mid=\varepsilon\} & \text { if } t \in \mathbb{H}, \\ \left\{z \in \mathcal{F}_{N} \mid \operatorname{Im}\left(\sigma_{t} z\right)=\frac{1}{\varepsilon}\right\} & \text { if } t \text { is a cusp of } \Gamma_{0}(N) .\end{cases}
$$

Then

$$
\partial \mathcal{F}_{N}(g, \varepsilon)=\bigcup_{t \in S(g) \cup \mathcal{C}_{N}} \gamma(t, \varepsilon)
$$


For $t \in \mathbb{H}$ let $\operatorname{Res}_{t}(g)$ be the residue of $g$ at $t$ on $\mathbb{H}$. Using the argument of Lemma 3.1 and Proposition 3.5 in [5], we prove the following lemma.

Lemma 3.1. Let $g(z):=\sum_{n=0}^{\infty} a(n) q^{n}$ be a meromorphic modular form of weight 2 on $\Gamma_{0}(N)$. Suppose that $g(z)$ is holomorphic at each cusp and that every pole of $g(z)$ is a simple pole. Then we have

$$
\begin{aligned}
\lim _{\varepsilon \rightarrow 0} \int_{\mathcal{F}_{N}(g, \varepsilon)} g(z) \cdot \xi_{0}\left(j_{N, m}(z)\right) d x d y \\
\quad=b_{m}(1,0) a(0)+a(m)+\sum_{t \in \mathcal{C}_{N}^{*}} \alpha_{t} g(t) j_{N, m}(t)+\sum_{t \in S(f)} \frac{2 \pi i}{l_{t}} \operatorname{Res}_{t}(g) j_{N, m}(t) .
\end{aligned}
$$

Proof. Note that

$$
d\left(g \cdot \overline{j_{N, m}} d z\right)=\bar{\partial}\left(g \cdot \overline{j_{N, m}} d z\right)=g \cdot \overline{\frac{\partial}{\partial \bar{z}} j_{N, m}} d \bar{z} d z=g(z) \cdot \xi_{0}\left(j_{N, m}\right)(z) d x d y .
$$

Thus, by Stokes' Theorem, we have

$$
\begin{aligned}
\int_{\mathcal{F}_{N}(g, \varepsilon)} g & (z) \cdot \xi_{0}\left(j_{N, m}(z)\right) d x d y \\
& =\sum_{t \in S(g)} \frac{1}{l_{\tau}} \int_{\gamma(t, \varepsilon)} g(z) j_{N, m}(z) d z+\sum_{t \in C_{N}} \int_{\gamma(t, \varepsilon)} g(z) j_{N, m}(z) d z .
\end{aligned}
$$

Note that if $t \in \mathcal{C}_{N}$ and $\varepsilon$ is sufficiently small, then

$$
\int_{\gamma(t, \varepsilon)} g(z) j_{N, m}(z) d z=\alpha_{t} \int_{-\frac{1}{2}}^{\frac{1}{2}} g\left(\sigma_{t}^{-1}\left(x+i \frac{1}{\varepsilon}\right)\right) j_{N, m}\left(\sigma_{t}^{-1}\left(x+i \frac{1}{\varepsilon}\right)\right) d x .
$$

Here, $\alpha_{t}$ is a non-zero constant, and $\alpha_{t}=1$ for a cusp $t \sim \infty$.

Following the argument of Proposition 3.5 in [5], for a cusp $t \sim \infty$ we have

$$
\lim _{\varepsilon \rightarrow 0} \int_{\gamma(t, \varepsilon)} g(z) j_{N, m}(z) d z=b_{m}(1,0) a(0)+a(m)
$$

and for $t \not \infty$,

$$
\lim _{\varepsilon \rightarrow 0} \int_{\gamma(t, \varepsilon)} g(z) j_{N, m}(z) d z=\alpha_{t} g(t) j_{N, m}(t) .
$$

From now on, we consider

$$
\lim _{\varepsilon \rightarrow 0} \int_{\gamma(t, \varepsilon)} g(z) j_{N, m}(z) d z
$$

for $t \in S(g)$. Suppose that $g(z)$ has the Laurent series at $t$ :

$$
g(z)=\sum_{n=-1}^{\infty} a_{n}(t)(z-t)^{n} .
$$


Then we have

$$
\begin{aligned}
\int_{\gamma(t, \varepsilon)} g(z) j_{N, m}(z) d z & =\frac{1}{l_{\tau}} \int_{0}^{1} g\left(t+\varepsilon e^{2 \pi i u}\right) j_{N, m}\left(t+\varepsilon e^{2 \pi i u}\right) 2 \pi i \varepsilon e^{2 \pi i u} d u \\
& =\frac{2 \pi i}{l_{\tau}} \int_{0}^{1}\left(\sum_{n=0}^{\infty} a_{n-1}(t)\left(\varepsilon e^{2 \pi i u}\right)^{n}\right) j_{N, m}\left(t+\varepsilon e^{2 \pi i u}\right) d u \\
& =\frac{2 \pi i}{l_{\tau}} \sum_{n=0}^{\infty} a_{n-1}(t) \varepsilon^{n} \int_{0}^{1} e^{2 \pi i n u} j_{N, m}\left(t+\varepsilon e^{2 \pi i u}\right) d u
\end{aligned}
$$

Thus, we get

$$
\begin{aligned}
\lim _{\varepsilon \rightarrow 0} \int_{\gamma(t, \varepsilon)} g(z) j_{N, m}(z) d z & =\lim _{\varepsilon \rightarrow 0} \frac{2 \pi i}{l_{\tau}} \sum_{n=0}^{\infty} a_{n-1}(t) \varepsilon^{n} \int_{0}^{1} e^{2 \pi i n u} j_{N, m}\left(t+\varepsilon e^{2 \pi i u}\right) d u \\
& =\frac{2 \pi i}{l_{\tau}} a_{-1}(t) \int_{0}^{1} j_{N, m}(t) d u=\frac{1}{l_{\tau}} 2 \pi i a_{-1}(t) j_{N, m}(t) \\
& =\frac{2 \pi i}{l_{\tau}} \operatorname{Res}(g) j_{N, m}(t) .
\end{aligned}
$$

This completes the proof.

\section{Proof of Theorem 1.1}

Suppose that $g$ is a meromorphic modular form of weight 2 on $\Gamma_{0}(N)$. For $\tau \in \mathbb{H}$, let $Q_{\tau}$ be the image of $\tau$ under the canonical map from $\mathbb{H} \cup \mathbb{Q} \cup\{\infty\}$ to $X_{0}(N)$. The residue of $g$ at $Q_{\tau}$ on $X_{0}(N)$, denoted by $\operatorname{Res}_{Q_{\tau}} g d z$, is well defined since we have the canonical correspondence between a meromorphic modular form of weight 2 on $\Gamma_{0}(N)$ and a meromorphic 1-form on $X_{0}(N)$. Suppose that $t$ is a cusp of $\Gamma_{0}(N)$. Let $\sigma_{t}^{*}$ be a matrix in $S L_{2}(\mathbb{Z})$ such that $\sigma_{t}^{*} \infty=t$. Then there exists $\alpha_{t}$ such that

$$
\sigma_{t}^{*-1} \Gamma_{0}(N)_{t} \sigma_{t}^{*}=\left\{ \pm\left(\begin{array}{cc}
1 & k \alpha_{t} \\
0 & 1
\end{array}\right) \mid k \in \mathbb{Z}\right\}
$$

where $\Gamma_{0}(N)_{t}$ denotes the stabilizer of the cusp $t$ in $S L_{2}(\mathbb{Z})$. For convenience we define $g(t)$ by the constant term of the Fourier expansion of $g(z)$ at $t$. If $\operatorname{Res}_{\tau} g$ denotes the residue of $g$ at $\tau$ on $\mathbb{H}$, then we obtain

$$
\begin{aligned}
& \operatorname{Res}_{Q_{\tau}} g d z=\frac{1}{l_{\tau}} \operatorname{Res}_{\tau} g \text { if } \tau \in \mathbb{H}, \\
& \operatorname{Res}_{Q_{t}} g d z=\alpha_{t} g(t) \text { if } t \in \mathcal{C}_{N} .
\end{aligned}
$$

Here, $l_{\tau}$ is the order of the isotropy group at $\tau$. In particular, if $f$ is a meromorphic modular form of weight $k$ on $\Gamma_{0}(N)$ and $g=\frac{\theta f}{f}$, then the residue of $g$ at each point on $X_{0}(N)$ is determined by the order of its zero or pole. If we denote by $\operatorname{ord}_{\tau}(f)$ the order of the zero or pole of $f$ at $\tau$ as a complex function on $\mathbb{H}$, then

$$
\nu_{\tau}^{(N)}(f)=\frac{1}{l_{\tau}} \operatorname{ord}_{\tau}(f)
$$

Note that the constant term of the Fourier expansion of $\frac{\theta f}{f}$ at a cusp $t$ is equal to the order of its zero or pole at the cusp. Thus, for each $t \in \mathbb{H} \cup \mathbb{Q} \cup\{\infty\}$ we have

$$
2 \pi i \cdot \operatorname{Res}_{Q_{t}} \frac{\theta f}{f}=\nu_{t}^{(N)}(f) .
$$

Proof of Theorem 1.1. We begin by stating a lemma which was proved by Eholzer and Skoruppa in 9 . 
Lemma 4.1 (9]). Suppose that $f=\sum_{n=h}^{\infty} a(n) q^{n}$ is a meromorphic modular function in a neighborhood of $q=0$ and that $a(h)=1$. Then there are uniquely determined complex numbers $c(n)$ such that

$$
f=q^{h} \prod_{n=1}^{\infty}\left(1-q^{n}\right)^{c(n)},
$$

where the product converges in a small neighborhood of $q=0$. Moreover, the following identity is true:

$$
\frac{\theta f}{f}=h-\sum_{n \geq 1} \sum_{d \mid n} c(d) d q^{n}
$$

Recall that

$$
f_{\theta}(z)=\frac{\theta f(z)}{f(z)}+\frac{k / 12-h}{N-1} \cdot N E_{2}(N z)+\frac{h-N k / 12}{N-1} \cdot E_{2}(z) .
$$

The function $f_{\theta}(z)$ is a meromorphic modular form of weight 2 on $\Gamma_{0}(N)$. Note that $f_{\theta}(z)$ is holomorphic at each cusp of $\Gamma_{0}(N)$. Moreover, the function

$$
\frac{k / 12-h}{N-1} \cdot N E_{2}(N z)+\frac{h-N k / 12}{N-1} \cdot E_{2}(z)
$$

is holomorphic on $\mathbb{H}$. Thus, we have

$$
\operatorname{Res}_{\tau} f_{\theta}=\operatorname{Res}_{\tau} \frac{\theta f}{f}
$$

for $\tau \in \mathbb{H}$. Using Lemma 3.1 and (4.1), we complete the proof.

\section{Proofs of Theorems 1.4 and 1.7}

Proof of Theorem 1.4. Note that

$$
\begin{aligned}
f_{\theta}(z) & =\frac{\theta f(z)}{f(z)}+\frac{k / 12-h}{N-1} \cdot N E_{2}(N z)+\frac{h-N k / 12}{N-1} \cdot E_{2}(z) \\
& =\sum_{n=1}^{\infty}\left(-\sum_{d \mid n} c(d) d+\frac{2 N k-24 h}{N-1} \sigma_{1}(n)+\frac{24 h-2 k}{N-1} N \sigma_{1}(n / N)\right) q^{n} .
\end{aligned}
$$

This completes the proof by Theorem 1.1 .

Proof of Theorem [1.7. We follow the argument of Theorem 3 in [6] (or Theorem 4 in [2]). Suppose that $f(z)$ has the $q$-expansion of the form

$$
f(z):=q^{h}+\sum_{n=h+1}^{\infty} a(n) q^{n}=q^{h} \prod_{n=1}^{\infty}\left(1-q^{n}\right)^{c(n)}
$$

as in Lemma 4.1. Let

$$
b(n):=\sum_{d \mid n} c(d) d
$$

Lemma 4.1 implies that

$$
\left(q^{h}+\sum_{n=h+1}^{\infty} a(n) q^{n}\right)\left(h-\sum_{n=1}^{\infty} b(n) q^{n}\right)=h q^{h}+\sum_{n=h+1}^{\infty} n a(n) q^{n} .
$$


Thus, for $n \geq 1$ we have

$$
n a(h+n)=-b(1) a(h+n-1)-b(2) a(h+n-2)-\cdots-b(n) .
$$

From this recurrence we obtain

$$
\begin{aligned}
& b(n)=-n a(h+n) \\
& n \cdot \sum_{\substack{m_{1}+2 m_{2}+\cdots+(n-1) m_{n-1}=n \\
m_{1}, \cdots, m_{n-1} \geq 0}}(-1)^{m_{1}+\cdots+m_{n-1}} \frac{\left(m_{1}+\cdots+m_{n-1}-1\right) !}{m_{1} ! m_{2} ! \cdots m_{n-1} !} a(h+1)^{m_{1}} \cdots \\
& a(h+n-1)^{m_{n-1}}
\end{aligned}
$$

(see (2.11) and Example 20 in $\S 1.2$ of [12]). Note that by Theorem 1.1.

$$
\begin{aligned}
b(n) & =\sum_{\tau \in \mathcal{F}_{N} \cup \mathcal{C}_{N}^{*}} \nu_{\tau}^{(p)}(f(z)) j_{N, m}(\tau)-\int_{\mathcal{F}_{N}}^{r e g} f_{\theta}(z) \cdot \xi_{0}\left(j_{N, m}(z)\right) d x d y \\
& +\frac{2 N k-24 h}{N-1} \sigma_{1}(m)+\frac{24 h-2 k}{N-1} N \sigma_{1}(m / N) .
\end{aligned}
$$

This completes the proof.

\section{ACKNOWLEDGEMENTS}

This work was supported by the Korea Research Foundation Grant funded by the Korean Government (KRF-2008-331-C00005), and the author wishes to express his gratitude to KIAS for its support through the Associate Membership program.

The author also thanks the referee for useful comments.

\section{REFERENCES}

[1] M. Abramowitz, I. Stegun, Pocketbook of Mathematical Functions, Verlag Harri Deutsch, Thun, 1984. MR768931 (85j:00005b)

[2] S. Ahlgren, The theta-operator and the divisors of modular forms on genus zero subgroups, Math. Res. Lett. 10 (2003), no. 5-6, 787-798. MR2024734 (2004m:11059)

[3] R. E. Borcherds, Automorphic forms on $\mathcal{O}_{s+2,2}(\mathbb{R})$ and infinite products, Invent. Math. 120 (1995), no. 1, 161-213. MR1323986 (96j:11067)

[4] J. H. Bruinier, Borcherds products on $O(2, l)$ and Chern classes of Heegner divisors, Lect. Notes Math. 1780, Springer-Verlag, Berlin (2002). MR.1903920 (2003h:11052)

[5] J. H. Bruinier, J. Funke, On two geometric theta lifts, Duke Math. J. 125 (2004), no. 1, 45-90. MR2097357 (2005m:11089)

[6] J. Bruinier, W. Kohnen, K. Ono, The arithmetic of the values of modular functions and the divisors of modular forms, Compos. Math. 140 (2004), no. 3, 552-566. MR2041768 (2005h:11083)

[7] J. H. Bruinier, T. Yang, Twisted Borcherds products on Hilbert modular surfaces and their CM values, Amer. J. Math. 129 (2007), no. 3, 807-841. MR.2325105 (2008f:11057)

[8] D. Choi, On values of a modular form on $\Gamma_{0}(N)$, Acta Arith. 121 (2006), no. 4, 299-311. MR2224397 (2006m:11051)

[9] W. Eholzer, N.-P. Skoruppa, Product expansions of conformal characters, Phys. Lett. B 388 (1996), no. 1, 82-89. MR1418608 (97k:81132)

[10] D. Elkies, The existence of infinitely many supersingular primes for every elliptic curve over $Q$, Invent. Math. 89 (1987), no. 3, 561-567. MR903384(88i:11034)

[11] D. A. Hejhal, The Selberg Trace Formula for PSL(2,R), Lecture Notes in Mathematics 1001, Springer-Verlag (1983). MR711197(86e:11040) 
[12] G. Macdonald, Symmetric functions and Hall polynomials, Second Edition, Oxford University Press, Oxford, 1995. MR 1354144 (96h:05207)

[13] D. Niebur, A class of nonanalytic automorphic functions, Nagoya Math. J. 52 (1973), 133145. MR0337788(49:2557)

School of Liberal Arts and Sciences, Korea Aerospace University, 200-1, HwajeonDONG, Goyang, GyeongGi 412-791, Korea

E-mail address: choija@kau.ac.kr 